\title{
Aplicação do método GUM para verificação da calibração de paquímetros de um laboratório de metrologia
}

\section{Application of the GUM method to check the calibration of calipers in a metrology laboratory}

\author{
Isadora Maria Martins ${ }^{1}$, Gabriel Inácio Pontin ${ }^{1}$ \\ ${ }^{1}$ Centro Universitário da Fundação Educacional de Barretos, Unifeb, Barretos, SP.
}

\begin{abstract}
Resumo
No estudo de metrologia tem-se que o cálculo de incerteza demonstra qual a precisão do instrumento de medição, possibilitando ao usuário a escolha do procedimento de calibração adequado. Uma das maneiras de se verificar a veracidade das informações é utiliza métodos regulamentados através dos padrões existentes por meio das normativas oferecidas em todo o país. Esse trabalho tem por objetivo demonstrar através do método estatístico com representação numérica e gráfica a medidas, erros e incertezas presentes nos paquímetros disponíveis no laboratório de metrologia da instituição de ensino. Através dos resultados pode se verificar que o método escolhido foi conforme o esperado, já que se estimava que houvesse erro entre os paquímetros para que as análises matemáticas finais demonstraram a veracidade dos dados.
\end{abstract}

Palavras-chave: calibração; medida; paquímetro; incertezas

\begin{abstract}
The metrology study shows that the uncertainty calculation demonstrates the accuracy of the measurement instrument, thus enabling the user to choose the appropriate calibration procedure. One of the ways to verify the veracity of the information is to use methods regulated by the existing standards through the regulations offered throughout the country. This work aims to demonstrate, through the statistical method with numerical and graphical representation, the measures, errors, and uncertainties present in the calipers available in the metrology laboratory of the educational institution. Through the results, we can verified that the chosen method was as expected, since it was estimated that there was an error between the calipers so that mathematical analyzes demonstrated the veracity of the data.
\end{abstract}

Keywords: calibration; measure; calipers; uncertainties

\footnotetext{
Autor correspondente: Isadora Maria Martins- Centro Universitário da Fundação Educacional de Barretos, Unifeb - Av. Prof Roberto Frade Monte, 386, Barretos, SP, Brasil - CEP:14783-226 - E-mail: isadoraengmecanica@gmail.com
}

Recebido para publicação: 08/12/2019

Aceito para publicação: 07/05/2020

https://doi.org/10.4322/1980-0029.172020 


\section{Introdução}

Os resultados das medições dos ensaios e das análises também não podem ser perfeitos. Uma das principais tarefas de um experimentador é identificar as fontes de erro que podem afetar o processo de medição e quantificar tais fontes de erro de acordo com padrões existentes.

Essa variação de elementos mensurados é designada, atualmente, por incerteza. A palavra "erro", que durante anos foi utilizada com esse mesmo significado, está hoje em dia reservada para designar o afastamento entre o valor obtido numa medição e o correspondente valor verdadeiro, o qual é, em geral, desconhecido (Cabral, 2004).

O Guia para Expressão da Incerteza de Medição da ISO (International Organization for Standardization) também conhecido como GUM, é um método oficial publicado pela ISO, em conjunto com o BIPM (Bureau international des poids et mesures) e outras entidades internacionais da área científica, que estabelece uma forma de cálculo de incerteza de forma que possa ser universalmente aplicada (Associação Brasileira de Normas Técnicas, 2012).

O ISO GUM 95, além de ser um documento para a utilização mundial da estimativa da incerteza de um resultado de medição, se estabelece também como uma literatura balizadora de diversas outras análises, interpretações e aplicações. Uma destas interessantes interpretações do guia ocorre quando ele se estabelece como um documento orientado para a análise e definição da especificação de uma instrumentação adequada à tolerância de um determinado processo (Couto \& Monteiro, 2000).

O GUM foi elaborado no sentido de harmonizar as metodologias utilizadas pelos laboratórios de metrologia para a estimativa da incerteza nas medições bem como servir como um guia de fácil entendimento e implementação nas diferentes áreas da metrologia (Associação Brasileira de Normas Técnicas, 2012).

Diversos fatores justificam a importância do cálculo da incerteza. Por exemplo, quando são realizados ensaios para estabelecer a conformidade de um produto frente a uma especificação ou legislação, é importante para as partes interessadas (comprador, fornecedor, organismo regulamentador, entre outros.) que a incerteza seja tomada em consideração (European Co-Operation for Accreditation, 2003).
O paquímetro é um instrumento de medida muito utilizado na prática industrial. Ele é constituído essencialmente de uma parte metálica com escala, duas pontas apalpadoras (bicos de medição) que garantem o devido contato com a peça sob medição e um cursor com escala auxiliar para obter leituras fracionárias da menor divisão da escala principal. Os principais fabricantes de paquímetros oferecem uma variada gama desses instrumentos em função das possibilidades de combinação de princípios construtivos, tipos fundamentais de medição e aplicações específicas. Assim, além dos paquímetros com nônio, existem outros nos quais as leituras fracionárias são efetuadas num relógio mecânico ou a leitura completa num indicador digital (Volnei, 2001)

Justifica-se que devido à grande utilização recorrente nas aulas em laboratórios, os paquímetros podem gerar erros por perderem a precisão e, com isso, prejudicar até mesmo perda de peças por medidas fora das determinações necessárias nos laboratórios de usinagem e também podendo ser um fator prejudicial ao ensino dos alunos, principalmente dos anos iniciais, para melhor entendimento do uso e das medidas. Daí a importância de analisar e demostrar a incerteza entre os paquímetros do laboratório de metrologia de uma instituição de ensino, de forma numérica e gráfica, demonstrando suas falhas em medidas, seguindo as definições da norma ISO GUM 95 e mostrando que eles podem variar as medidas fixas e fornecer dados incertos. Juntamente com o desvio padrão comumente utilizado para medir a confiabilidade de cálculos estatísticos, permitindo sintetizar os resultados de uma experiência repetidas vezes, sendo utilizado para medir a dispersão de valores individuais em torno da média nada mais é a raiz quadrada da variância, pode ser usado para descrever a quantidade de dispersão na distribuição da frequência.

\section{Materiais e métodos}

Para análise da incerteza usaram-se seis paquímetros, que são os utilizados e únicos disponíveis no laboratório institucional, uma peça de madeira (Figura 1) e um software de planilhas eletrônicas, para plotagem de gráfico e análise da linearidade dos dados.

O material da peça escolhida foi madeira, devido ao seu coeficiente de dilatação térmica ser menor em relação ao aço quando trabalhados em baixas temperaturas. Sendo assim, sofreria 


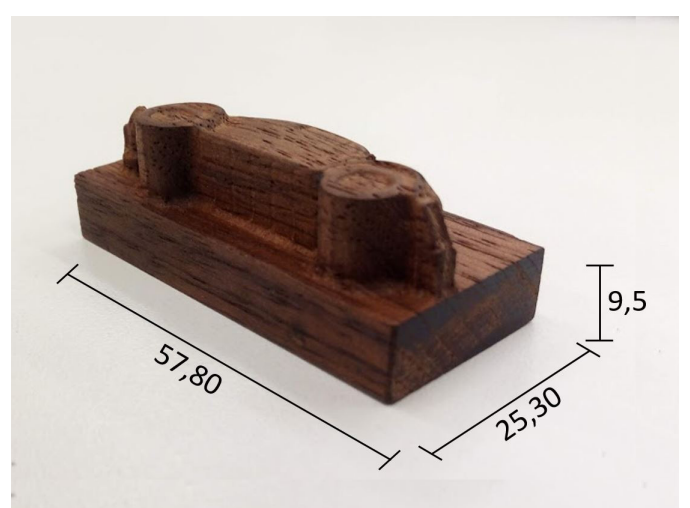

Figura 1. Peça usinada utilizada para análise com medidas em $\mathrm{mm}$.

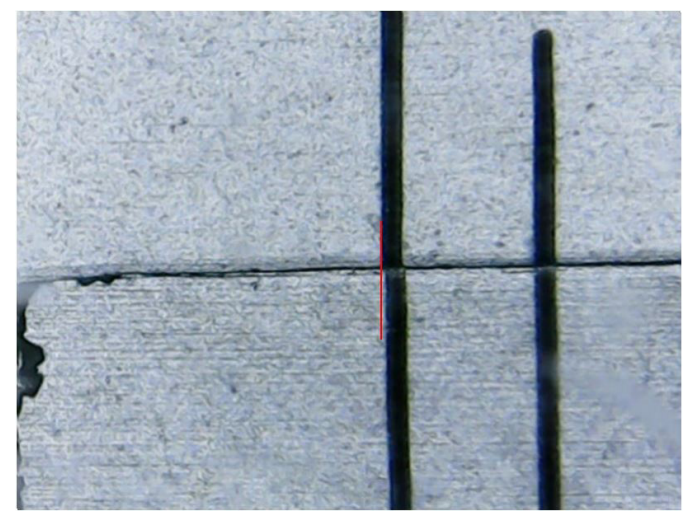

Figura 2. Precisão do paquímetro analisada com zoom de 46x.

menor variação ao ser manipulado em temperatura de ambiente controlado à $23^{\circ} \mathrm{C}$ e minimizaria o erro do operador.

A peça de madeira tem as dimensões fornecidas pelo programa de modelagem 3D para criação de peças (suas dimensões estão disponíveis na Figura 1), sua largura varia entre 24,90 a $26,00 \mathrm{~mm}$, para que na sua análise o método com 12 pontos escolhidos ao longo da peça no processo de usinagem, sendo fabricado no laboratório de mecânica 1 , mostrado na Figura 1.

Para verificação da variação de largura ao longo do corpo foram escolhidos 12 pontos aleatórios e demarcados de acordo com estudos de amostragem da área útil do corpo de prova, sendo utilizado um paquímetro com precisão de $0,02 \mathrm{~mm}$, em que a mesma amostragem foi inspecionada através de um microscópio USB da marca Electronic Magnifier ${ }^{\circledR}$ em um zoom de 46x antes e após as medidas para verificação exata na sua precisão, como mostra a Figura 2.

\section{Método de GUM}

O GUM em harmonia com os métodos utilizados levanta a estimativa de incerteza de medições. Essas incertezas podem ser analisadas em 2 tipos mais comuns: tipo A e tipo $\mathrm{B}$.

A incerteza padrão tipo A deve ser baseada em métodos estatísticos válidos para tratamento de dados.

A incerteza padrão tipo B é efetuada por outros meios que não a análise estatística de uma série de observações. É usualmente baseada em julgamentos científicos utilizando todas as informações disponíveis.

O procedimento de estimativa de incerteza nas medições começa na determinação dos coeficientes demonstrados na Equação 1, com a determinação da incerteza combinada, o número de graus de liberdade e o fator de abrangência e incerteza expandida, Equação 2.

$$
\mathrm{uc}=\sqrt{\left(\sum[\mathrm{ui}(\mathrm{y}) \wedge 2]\right)}
$$

Sendo:

uc: é a incerteza padrão combinada, ou simplesmente incerteza combinada;

ui(y) são as contribuições de incerteza para o mensurando $\mathrm{y}$.

$$
\mathrm{U}=\mathrm{K} * \mathrm{uc}(\mathrm{y})
$$

Sendo:

U: é a incerteza expandida;

$\mathrm{K}$ : fator de abrangência de incerteza.

Para obter o valor de ui, há dois métodos, tipo a e b, em que:

$\mathrm{ui}=\sqrt{(1 /(\mathrm{n}-1)) *(\Sigma(\mathrm{xi}) \wedge 2) / \mathrm{n}}$

Sendo:

xi: amostra individual;

n: número de análises.

ua $=$ ui $/(\sqrt{\mathrm{n}})$

$\mathrm{ub}=\mathrm{st} /(\sqrt{3})$

Sendo:

st: é a precisão do material dividido por 2.

\section{Desvio Padrão}

O símbolo para o desvio padrão em um conjunto de dados observados é "s".

$\mathrm{s}=\sqrt{(([(\mathrm{x}-[\mathrm{xi}] \wedge 2)]) /(\mathrm{n}-1))}$ 


\section{Análise Diagonal}

Essa análise tem por objetivo demonstrar através de uma reta qual o caminho que todas as leituras devem seguir.

Para que seja feita a análise, deve-se relembrar o estudo da geometria analítica, a equação fundamental da reta, que está demostrada na Equação 7. Outra forma de representar simplificadamente é analisando a inclinação da reta e o coeficiente angular.

$$
\mathrm{y}-\mathrm{y} 0=\mathrm{m} \cdot(\mathrm{x}-\mathrm{x} 0)
$$

\section{Resultados}

Com os dados práticos obtidos em laboratório durante análise foi possível montar tabelas de valores para plotagem de gráficos e analisar os possíveis erros de medidas dos paquímetros.

Observa-se que essas são as medidas nos pontos determinados de acordo com os dados obtidos pelo software de dados (Tabela 1).

Tabela 1. Medidas da peça fornecidas pelo software 3D em mm

\begin{tabular}{cc}
\hline Medida & Dados do Software \\
\hline 1 & 24,94 \\
2 & 25,04 \\
3 & 25,14 \\
4 & 25,24 \\
5 & 25,34 \\
6 & 25,44 \\
7 & 25,54 \\
8 & 25,64 \\
9 & 25,74 \\
10 & 25,84 \\
11 & 25,94 \\
12 & 26,04 \\
\hline
\end{tabular}

Com a verificação do dado que foi fornecido pelo software, pode-se observar a diferença de medidas em relação aos outros paquímetros (Tabela 2).

Após isso, pode-se encontrar a média de medidas, o desvio padrão por amostra e observar a diferença de medidas entre a incerteza combinada e expandida de cada um.

Analisando todos os dados, nota-se na Tabela 2 a desproporcionalidade individual de cada medida variando de um paquímetro para outro, gerando uma enorme diferença entre pontos, isso também ocorre ao longo de todos os pontos em geral.

Percebe-se que a incerteza do tipo B só é mais confiável no paquímetro 5. Já a incerteza combinada (ui), apesar de ter um bom grau de confiabilidade $(95,2 \%)$, ainda não atingiu o valor mínimo de cada paquímetro, devido às variáveis que envolvem as análises (Tabela 3).

Para realizar a análise de erro e encontrar os pontos perfeitos entre os paquímetros, foi feita a análise diagonal dos paquímetros.

Nota-se na Figura 3 que a diagonal passa entre alguns pontos do gráfico. E reta mostra a estatística de qual seria a reta ideal para todos os paquímetros em comparação com os dados fornecidos pelo software $3 \mathrm{D}$.

\section{Discussão}

Em geral, nas análises numéricas nota-se uma diferença entre paquímetros sobre as medidas com uma variação entre pontos. Analisando através das incertezas pode-se notar que esse erro é gradualmente alto, visto que mesmo com o maior grau de confiabilidade, sua maior incerteza não atingiu o valor menor entre pontos dentre todos os paquímetros.

Já na plotagem gráfica, esperava-se que todos os paquímetros formassem um mesmo tipo de

Tabela 2. Dados fornecidos pelos paquímetros em $\mathrm{mm}$.

\begin{tabular}{ccccccc}
\hline Medida & Paquímetro 1 & Paquímetro 2 & Paquímetro 3 & Paquímetro 4 & Paquímetro 5 & Paquímetro 6 \\
\hline 1 & 25,45 & 25,40 & 25,05 & 25,25 & 24,94 & 25,49 \\
2 & 25,46 & 25,41 & 25,10 & 25,27 & 25,04 & 25,50 \\
3 & 25,47 & 25,42 & 25,15 & 25,29 & 25,14 & 25,51 \\
4 & 25,48 & 25,43 & 25,20 & 25,31 & 25,24 & 25,52 \\
5 & 25,49 & 25,44 & 25,25 & 25,33 & 25,34 & 25,53 \\
6 & 25,50 & 25,45 & 25,30 & 25,35 & 25,44 & 25,54 \\
7 & 25,51 & 25,46 & 25,35 & 25,37 & 25,54 & 25,55 \\
8 & 25,52 & 25,47 & 25,40 & 25,39 & 25,64 & 25,56 \\
9 & 25,53 & 25,48 & 25,45 & 25,41 & 25,74 & 25,57 \\
10 & 25,54 & 25,49 & 25,50 & 25,43 & 25,84 & 25,58 \\
11 & 25,55 & 25,50 & 25,55 & 25,45 & 25,94 & 25,59 \\
12 & 25,56 & 25,51 & 25,60 & 25,47 & 26,04 & 25,60 \\
\hline
\end{tabular}


Tabela 3. Dados pós análises em mm

\begin{tabular}{ccccrrr}
\hline \multirow{2}{*}{ Resultados em mm } & Paquímetro & Paquímetro & Paquímetro & Paquímetro & Paquímetro & Paquímetro \\
& $\mathbf{1}$ & $\mathbf{2}$ & $\mathbf{3}$ & $\mathbf{4}$ & $\mathbf{5}$ & $\mathbf{6}$ \\
\hline Média & 25,51 & 25,46 & 25,33 & 25,36 & 25,49 & 25,55 \\
Desvio Padrão & 0,04 & 0,04 & 0,18 & 0,07 & 0,36 & 0,04 \\
Incerteza Combinada (ui) & 26,64 & 26,59 & 26,45 & 26,49 & 26,63 & 26,68 \\
Incerteza Tipo A (ua) & 7,69 & 7,67 & 7,64 & 7,65 & 7,69 & 7,70 \\
Incerteza Tipo B (ub) & 0,01 & 0,01 & 0,01 & 0,01 & 0,06 & 0,01 \\
Incerteza Expandida (uc) & 23,07 & 23,02 & 22,91 & 22,94 & 23,06 & 23,11 \\
\hline
\end{tabular}

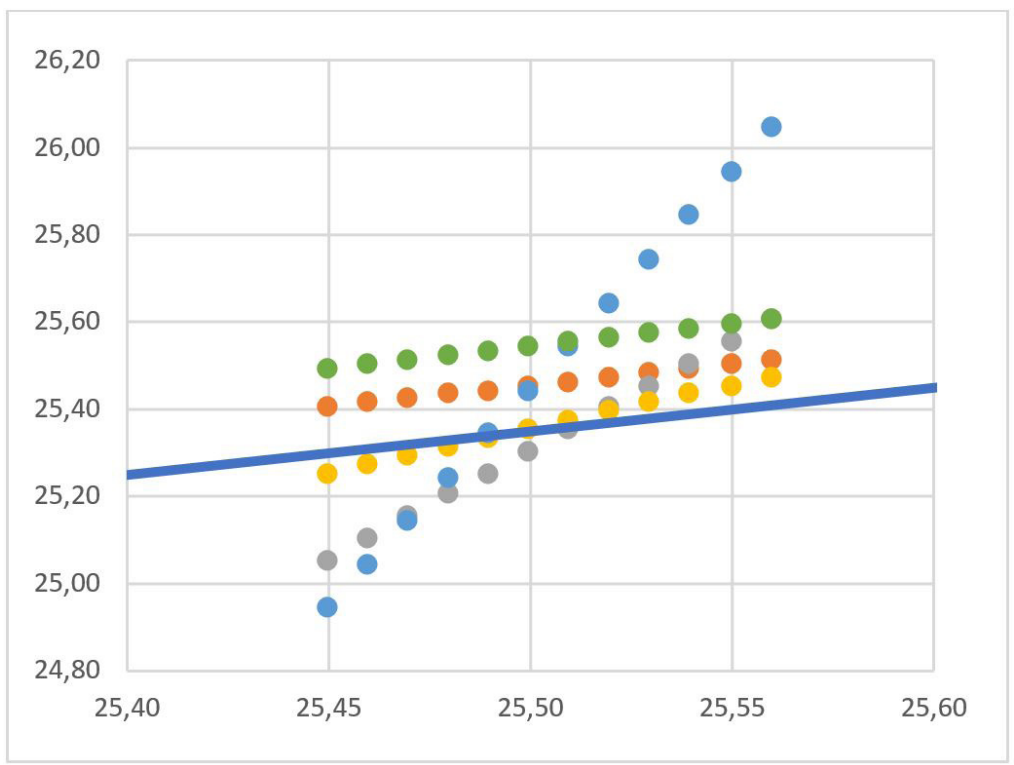

Figura 3. Análise diagonal de erro em mm.

reta, porém isso não ocorreu. Com a análise diagonal entre médias esperava-se que todos os paquímetros seguissem os mesmos pontos ao longo das análises.

\section{Conclusão}

Os resultados obtidos em laboratório atingiram o que se esperava, já que todos os paquímetros apresentaram erro, mesmo que pequeno, e era essencial que todos os cálculos fossem feitos e analisados demonstrando entre eles as suas diferenças. Para melhorar o uso desses equipamentos pelos alunos, o ajuste e calibração seria o melhor a se fazer, mas isso deve ser feito por um profissional capacitado e certificado, seguindo a norma ISO 9001:2015.

\section{Referências}

Associação Brasileira de Normas Técnicas - ABNT, \& Instituto Nacional de Metrologia, Normalização e Qualidade Industrial - INMETRO. (2012).
Guia para a Expressão da Incerteza de Medição. Primeira edição da primeira edição do BIPM de 2008. Rio de Janeiro: ABNT/INMETRO.

Cabral, P. (2004). Erros e incerteza nas medições (116 p.). Cidade: editora. Apostila de Circuitos Elétricos. Porto - PT: Editora IEP.

Couto, P. R. G., \& Monteiro, L. C. (2000). A Função de um Laboratório de Metrologia de acordo com o Contexto de Globalização. Metrologia 2000 (pp. 183-88). São Paulo: Editora INMETRO.

European Co-Operation for Accreditation EA. (2003). EA guidelines on the expression of uncertainty in quantitative testing. EA-4/16 G:2003. Recuperado em 20 de março de 2019, de www.european-accreditation.org

Volnei, A. (2001). Paquímetros (Vol. 1). Rio Grande do Sul: Rio Grande do Sul: Editora FURG. 